\title{
A SOFIC SYSTEM WITH INFINITELY MANY MINIMAL COVERS
}

\author{
SUSAN WILLIAMS
}

\begin{abstract}
We give an example of a sofic system $S$ together with an infinite collection of nonconjugate subshift of finite type covers of $S$ such that none of these covers factors through another subshift-of-finite-type cover to which it is not topologically conjugate.
\end{abstract}

1. Introduction. We answer a question of Boyle, Kitchens, and Marcus [BKM] concerning covers of sofic systems. A factor map between two symbolic systems is an onto, continuous shift-commuting map. A system which is a factor of a subshift of finite type (SFT) is called a sofic system. If $\pi$ is a factor map from a SFT $\Sigma$ to a sofic system $S$ we call $(\Sigma, \pi)$-or, for brevity, $\pi$-a cover of $S$.

If $(\Sigma, \pi)$ and $\left(\Sigma^{\prime}, \pi^{\prime}\right)$ are covers of $S$ we say $\pi$ factors through $\pi^{\prime}$, or $\pi^{\prime}$ intercepts $\pi$, if there is a factor map $\theta: \Sigma \rightarrow \Sigma^{\prime}$ for which

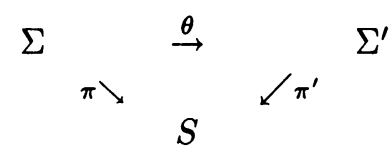

commutes. If $\theta$ is invertible (i.e., a topological conjugacy) we say $\pi$ and $\pi^{\prime}$ are conjugate over $S$. Note that this is stronger than saying $\pi$ and $\pi^{\prime}$ are topologically conjugate covers [BKM], which would require invertible $\theta$ and $\phi$ with

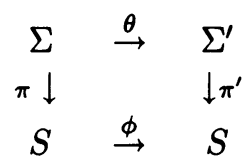

commuting. Departing from [BKM] we call a cover $\pi$ minimal if any $\pi^{\prime}$ which intercepts $\pi$ must be conjugate to $\pi$ over $S$.

For any sofic $S$, a well-known construction (see $[\mathbf{F}],[\mathbf{K}]$ ) gives the minimal past $\operatorname{cover}\left(\Sigma_{S}^{-}, \pi_{S}^{-}\right)$and minimal future cover $\left(\Sigma_{S}^{+}, \pi_{S}^{+}\right)$of $S$. These are indeed minimal in the sense defined above. $S$ is of almost finite type (AFT) if it has a cover $\pi$ which is one-to-one on a nontrivial open set. In [BKM] it was shown that $S$ is AFT if and only if it has a unique minimal cover (up to conjugacy over $S$ ); this minimal cover is necessarily conjugate to the minimal past and minimal future covers, and it intercepts every cover of $S$. If $S$ is not AFT, the minimal past and future covers are not conjugate over $S$.

Boyle, Kitchens, and Marcus ask the following question: If $S$ is not AFT, is there a finite collection $\left\{\pi_{1}, \ldots, \pi_{n}\right\}$ of covers such that every cover of $S$ factors

Received by the editors July $22,1985$.

1980 Mathematics Subject Classification (1985 Revision). Primary 54H20, 28D20, 05C20.

Work partly supported by a grant from the University of South Alabama Research Committee. 
through some $\pi_{i}$ ? We answer this question in the negative by exhibiting an infinite collection of minimal covers of a sofic $S$ of which no two are conjugate over $S$.

2. Example. We use the sofic $S$ described in [BKM]. $S$ is a system on the symbols $1,2,3,4$, and 5 obtained from the SFT $\Sigma$, given by the graph in Figure 1 , by a one-block map $\pi$ which identifies the states $3^{\mathrm{a}}, 3^{\mathrm{b}}$, and $3^{\mathrm{c}}$. The cover $\pi$ factors through the minimal past cover via a one-block map identifying states $3^{b}$ and $3^{\mathrm{c}}$; similarly, identifying $3^{\mathrm{a}}$ and $3^{\mathrm{b}}$ produces the minimal future cover (Figure 2). ( $\Sigma$ is simply the fibered product of $\Sigma_{S}^{-}$and $\Sigma_{S}^{+}$.)

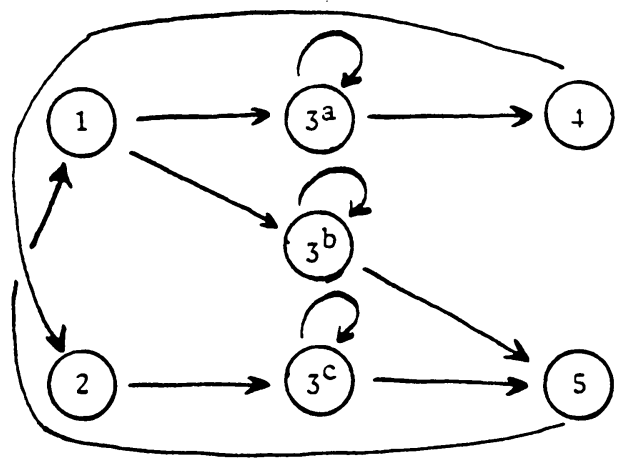

FIGURE 1
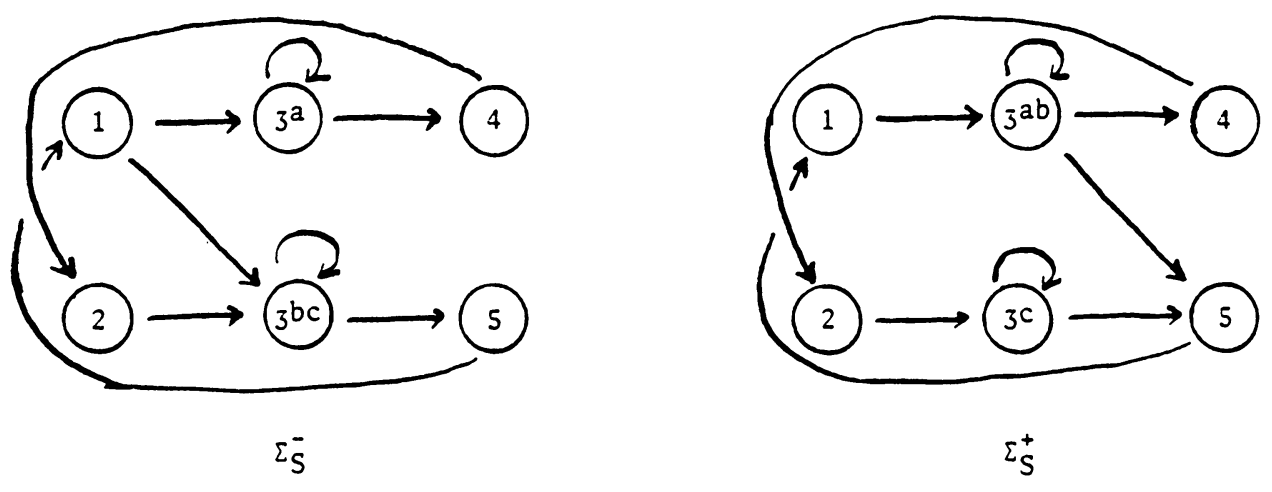

FIGURE 2

For each integer $n>1$ we first construct a cover $\left(\Gamma_{n}^{\prime}, \pi_{n}^{\prime}\right)$ of $S . \Gamma_{n}^{\prime}$ is given by the graph in Figure 3. The unlabeled states are arranged in $n$-cycles, and $\pi_{n}^{\prime}$ is the one-block map taking all these states to 3 . In effect we have separated out a "zero $\bmod n "$ component from each fixed point of $\Sigma$. Each $\pi_{n}^{\prime}$ factors through $\pi$ in an obvious fashion.

Finally, we obtain a cover $\left(\Gamma_{n}, \pi_{n}\right)$ by identifying corresponding states in the third and fifth $n$-cycles from the top in our diagram of $\Gamma_{n}^{\prime}$, and likewise identifying corresponding states in the second and fourth $n$-cycles (Figure 4). On the "zero $\bmod n "$ component we have mimicked the map which produces the minimal past cover from $\Sigma$, and on the remainder, that which gives the minimal future cover. 


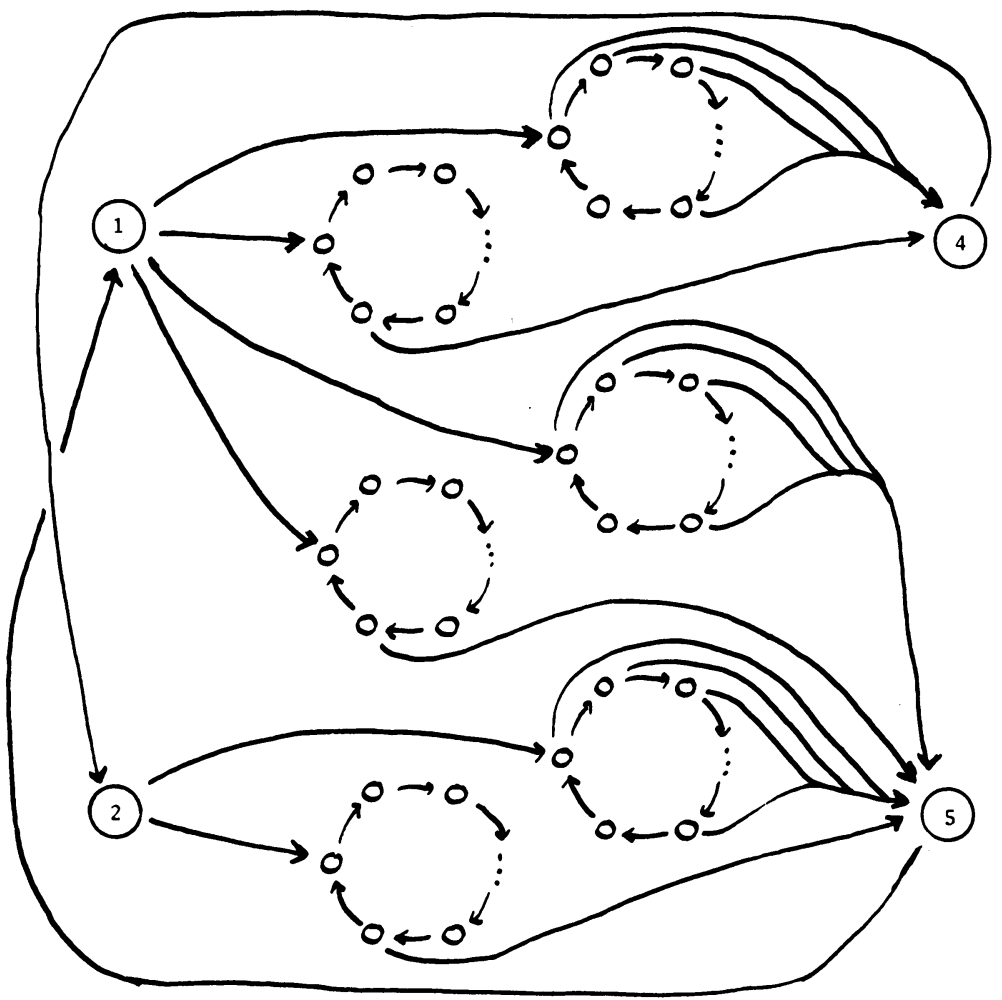

Figure 3

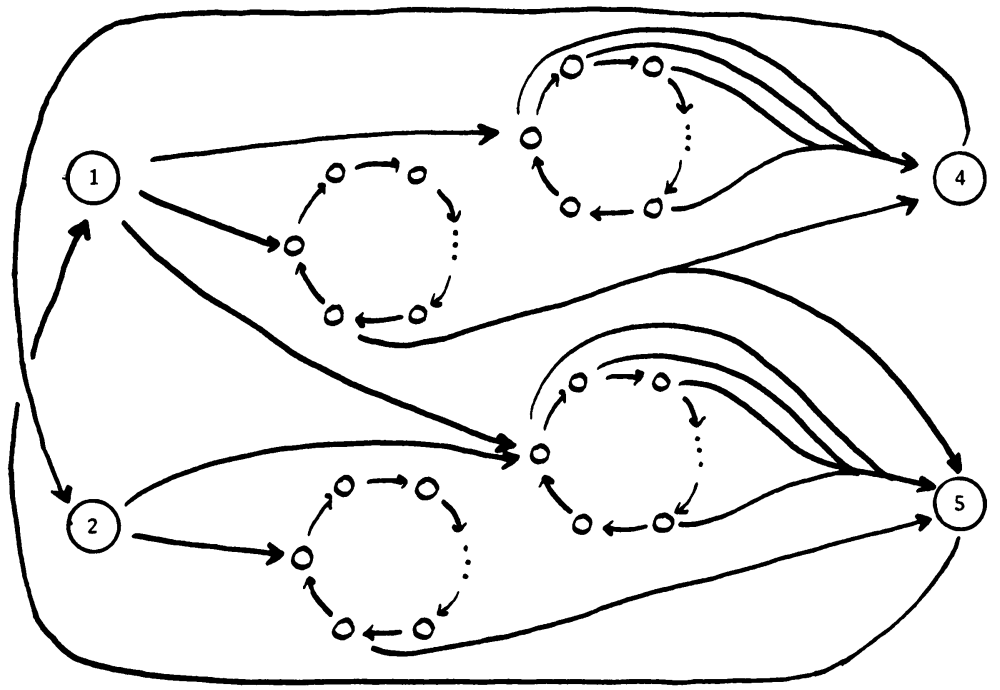

FIGURE 4 
For $m \neq n, \pi_{m}$ and $\pi_{n}$ are not topologically conjugate since they have periodic points of different periods. If we had $\pi_{n}=\Gamma \circ \theta$ with $\theta$ a noninvertible map taking $\Gamma_{n}$ to another SFT, $\theta$ would have to identify at least one pair of periodic points; we leave it to the reader to see that no such identification is possible.

\section{REFERENCES}

[BKM] M. Boyle, B. Kitchens and B. Marcus, A note on minimal covers for sofic systems, Proc. Amer. Math. Soc. 95 (1985), 403-411.

[F] R. Fischer, Graphs and symbolic dynamics, Proc. Colloq. Math. Soc. Janos Bolyai, Information Theory 16 (1975).

[K] W. Krieger, On sofic systems. I, Israel J. Math. 48 (1984), 305-330.

Department of Mathematics and Statistics, University of South Alabama, MOBILE, ALABAMA 36688 